\title{
Effect of head and limb orientation on trunk muscle activation during abdominal hollowing in chronic low back pain
}

\author{
Kevin Parfrey', Sean GT Gibbons², Eric J Drinkwater ${ }^{1,3}$ and David G Behm ${ }^{1 *}$
}

\begin{abstract}
Background: Individuals with chronic low back pain (CLBP) have altered activations patterns of the anterior trunk musculature when performing the abdominal hollowing manœuvre (attempt to pull umbilicus inward and upward towards the spine). There is a subgroup of individuals with CLBP who have high neurocognitive and sensory motor deficits with associated primitive reflexes (PR). The objective of the study was to determine if orienting the head and extremities to positions, which mimic PR patterns would alter anterior trunk musculature activation during the hollowing manoeuvre.
\end{abstract}

Methods: This study compared surface electromyography (EMG) of bilateral rectus abdominis (RA), external oblique (EO), and internal obliques (IO) of 11 individuals with CLBP and evident PR to 9 healthy controls during the hollowing manoeuvre in seven positions of the upper quarter.

Results: Using magnitude based inferences it was likely (>75\%) that controls had a higher ratio of left IO:RA activation with supine (cervical neutral), asymmetrical tonic neck reflex (ATNR) left and right, right cervical rotation and cervical extension positions. A higher ratio of right IO:RA was detected in the cervical neutral and ATNR left position for the control group. The CLBP group were more likely to show higher activation of the left RA in the cervical neutral, ATNR left and right, right cervical rotation and cervical flexion positions as well as in the cervical neutral and cervical flexion position for the right RA.

Conclusions: Individuals with CLBP and PR manifested altered activation patterns during the hollowing maneuver compared to healthy controls and that altering cervical and upper extremity position can diminish the group differences. Altered cervical and limb positions can change the activation levels of the IO and EO in both groups.

Keywords: Chronic low back pain, Exercise therapy, Motor control, Abdominal hollowing, Primitive reflexes, Stability exercise

\section{Background}

Over $80 \%$ of chronic low back pain (CLBP) occurrences are of unknown origin [1]. Many different treatment protocols have been used by physiotherapists and other clinicians [2]. One of the proposed reasons for the development of CLBP is an altered pattern of neuromuscular control of the spine [3-9]. There are numerous differences in the motor system when CLBP subjects are compared to normal subjects [10]. One finding is that the deep muscle system is impaired,

\footnotetext{
* Correspondence: dbehm@mun.ca

${ }^{1}$ School of Human Kinetics and Recreation, Memorial University of Newfoundland, St. John's, Newfoundland, Canada

Full list of author information is available at the end of the article
}

while the superficial muscles are variable with some displaying increased activity [9].

One treatment protocol for CLBP has been performing abdominal hollowing [4,11-14]. During a standardized exercise for abdominal hollowing, the activation of the larger superficial muscles such as the rectus abdominis (RA) are increased with CLBP patients [4]. The RA and external oblique (EO) muscles are global muscles responsible for gross movements of the trunk. The RA is the major trunk flexor while the EO are more responsible for lateral flexion and rotation [15]. It is theorized that the global muscles are substituting for the decreased amount of force, which the stabilizing muscles no longer

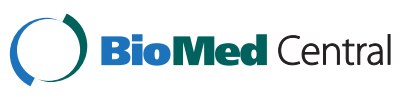


supply $[4,6]$. It is thought that if a specific exercise program is administered, which revolves around retraining the proper activation patterns during abdominal hollowing, that the altered pattern and function can be corrected [4]. The goal of this intervention is not to increase the strength of the abdominals, but rather to retrain the altered motor pattern of the abdominal musculature associated with CLBP.

While this type of treatment has been shown to be effective in treating CLBP patients by increasing function and decreasing pain levels [4], some patients have difficulty learning how to perform the hollowing maneuver. O'Sullivan et al. [16] reported that some individuals with CLBP took 4-5 weeks to properly learn and perform the hollowing maneuver, while Gibbons $[17,18]$ has identified a subgroup who cannot learn specific exercise and has recommended sub-classifying this group. This group is characterized by self reported neurocognitive, sensory motor, gross motor and respiratory deficits, as well as physical findings of sensory deficits and neurological soft signs (i.e. primitive reflexes : PR) [19]. Neurological soft signs are deviations in motor, sensory and integrative functions that do not signify localized brain dysfunction [20]. The presence of PR such as tonic neck and Moro reflexes within a CLBP population may indicate a deficiency in the supraspinal control of the anterior trunk muscles [17-19]. This may contribute to altered patterns of superficial muscle activity during abdominal hollowing [19].

PR are brain stem mediated complex automatic movement patterns which are evoked through touch or changes in body position $[21,22]$. The disappearance of PR are a sign of central nervous system (CNS) development as it indicates cortical inhibition, which is necessary for voluntary movement [23]. The presence of PR in adults have been associated with neurological disorders such as Alzheimer's and dementia $[24,25]$. It is also theorized that the recurrence of $\mathrm{PR}$ in adults may be an inherent consequence of usual aging [25]. As CLBP has been associated with atrophy of CNS gray matter, and specifically GABAergic inhibitory interneurons [26], then PR may resurface due to a decrease level of inhibition on the brainstem neurons responsible for the autonomic movement patterns. It may be possible that CLBP lead to alterations in the CNS and that the presence of PR is an indication of this change. Likewise this presence may explain why some individuals with CLBP have difficulty learning how to perform the hollowing maneuver. However, research is scant on this topic and more research is necessary to substantiate this possibility.

The objectives of this study were to examine if (1) there was a difference between the abdominal activation patterns of a CLBP group with apparent PR and a matched healthy control group when performing the hollowing maneuver; (2) by altering the position of the head and limbs to mimic that of a PR the CLBP group would have a similar activation pattern to the control; (3) there is a side specific activation pattern of the superficial abdominal muscles in either the CLBP group or the control group.

\section{Methods \\ Subjects}

Twenty participants (9 control and 11 CLBP) completed the experiment. Participants were split into a CLBP with prevalent PR group (Height: $163.6 \pm 9.1 \mathrm{~cm}$, Weight: $79.6 \pm 19 \mathrm{~kg}$, Age: $45.6 \pm 9.9$ years) or a control group without a history of CLBP (Height: $163.3 \pm 9.9 \mathrm{~cm}$, Weight: $78.8 \pm 15.3 \mathrm{~kg}$, Age: $42.3 \pm 8$ years). The control group was age, gender and mass matched to eliminate differences associated with different demographics and morphology. All subjects were explained the procedures of the study, given an opportunity to ask questions for clarification and made aware that they could stop the study at any point. All subjects were required to read and sign a consent form before participation. The Memorial University Human Investigation Committee approved the study (\#09.184).

Inclusion for the CLBP group was identified by a score of over 12 on the Rolland Morris Disability Questionnaire (RMDQ) [27] and suffering from low back pain for greater than 12 weeks [1]. Subjects were excluded from the CLBP group if there was a presence of severe postural abnormality and/or signs and symptoms of specific LBP including: radicular symptoms, radiological diagnosis (specifically spondylolisthesis or spondylolysis); limited neck range of motion or pain; known factors associated with primitive reflexes (severe postural abnormality, anti-depressant medication, opiate medication diabetes, previous neurological incidents, neurological conditions, heart surgery, diagnosed learning difficulties, withdrawal from alcohol or drug addictions or psychiatric conditions). A certified physiotherapist assessed the presence of PR. Intra-tester reliabilty of PR assessment has previously been established [28]. Exclusion criteria for the control group were any report of low back pain in the previous 6 months [29], limited neck range of motion or neck pain, or if they had any conditions (same as above) known to be associated with the presence of retained PR.

A novel approach was that an inclusion criterion for the CLBP group was the presence of at least one PR. PR presence was based on a $0-4$ rating scale from absent to the full pattern present [30]. If no remnant of the reflex was found, the rating was zero. If any aspect of the reflex pattern was present, it was deemed to be present and was rated then from 1-4 with 4 being the full reflex pattern. For assessment of the asymmetric tonic neck reflex (ATNR), the individual was placed in the supine position 
with the upper limb by the side. The ATNR was deemed present if active cervical rotation was accompanied by ipsilateral shoulder girdle elevation and/or the contralateral leg appears to shorten. For the secondary assessment of the ATNR, the individual stand with feet shoulder width apart and shoulders flexed to $90^{\circ}$. The ATNR was deemed present if active cervical rotation was followed by a weight shift to the side of rotation or ipsilateral shoulder girdle movement. For assessment of the stage 1 phase of the Moro reflex the individual was placed in the supine position with the glenohumeral joint in $30^{\circ}$ abduction and $90^{\circ}$ elbow flexion. The reflex was deemed present if active cervical extension to $30^{\circ}$ was followed by lumbar spine extension, hip extension or glenohumeral external rotation. Phase 2 of the Moro reflex was performed in supine crook lying. It was deemed present if active cervical flexion to $30^{\circ}$ was accompanied with posterior pelvic tilt, hip adduction or flexion, glenohumeral internal rotation or elbow flexion.

\section{Procedures}

\section{Electromyography}

The subjects were instructed to lie flat on a horizontal bench and were fitted bilaterally with surface electrodes on the RA, EO, and internal obliques (IO). All surface electrodes (Meditrace 130 ECG Conductive Adhesive $\mathrm{Ag} / \mathrm{AgCl}$ Electrodes, Tyco Healthcare Group LP, Mansfield, MA) were placed bilaterally on six different abdominal muscle sites. To reduce resistance of the signal, all sites for electrode placement were shaved, scrubbed with sand paper and rubbed with an alcohol-soaked paper towel [31-35]. This process removed body hair, dead skin cells, and oils [31-35]. Based on previously published articles from this laboratory, all electrodes were placed parallel to the muscle fibres, with an interelectrode difference of $2 \mathrm{~cm}$ [31-35]. The bilateral sites were the RA, which was defined as five centimetres below the xiphoid process and three centimetres lateral to the midline. The EO electrodes were placed five $\mathrm{cm}$ superior to the anterior superior iliac spine (ASIS) while the IO were placed immediately medial to the ASIS (Figure 1). All the described EMG sites have been used in a number of previous studies published from this laboratory [31-35]. The surface electrode site identified as IO may also detect EMG activity from the transversus abdominus (TrA) muscles as well [23-27]. A ground electrode for each of the six sites was placed over the iliac crest.

All EMG signals were collected over a 20 second period, sampled at $2000 \mathrm{~Hz}$ with a Blackman -61 band-pass filter between 10-500 Hz, and amplified (500X) (Biopac Systems MEC bi-polar differential 100 amplifier, Santa Barbara CA., input impedance $=2 \mathrm{M}$, common mode rejection ratio $>110 \mathrm{db} \min (50 / 60 \mathrm{~Hz})$, noise $>5 \mathrm{UV})$. EMG activity

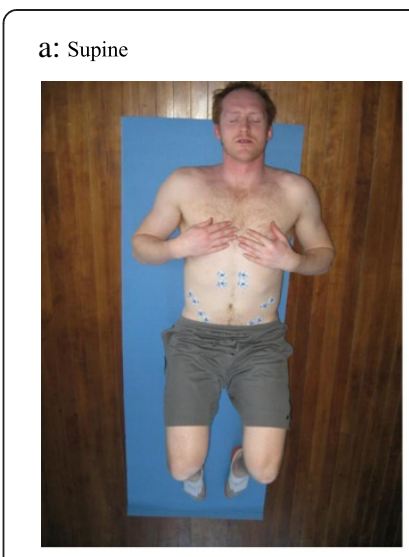

b: ATNR Left

c: Cervical Rotation to the Left
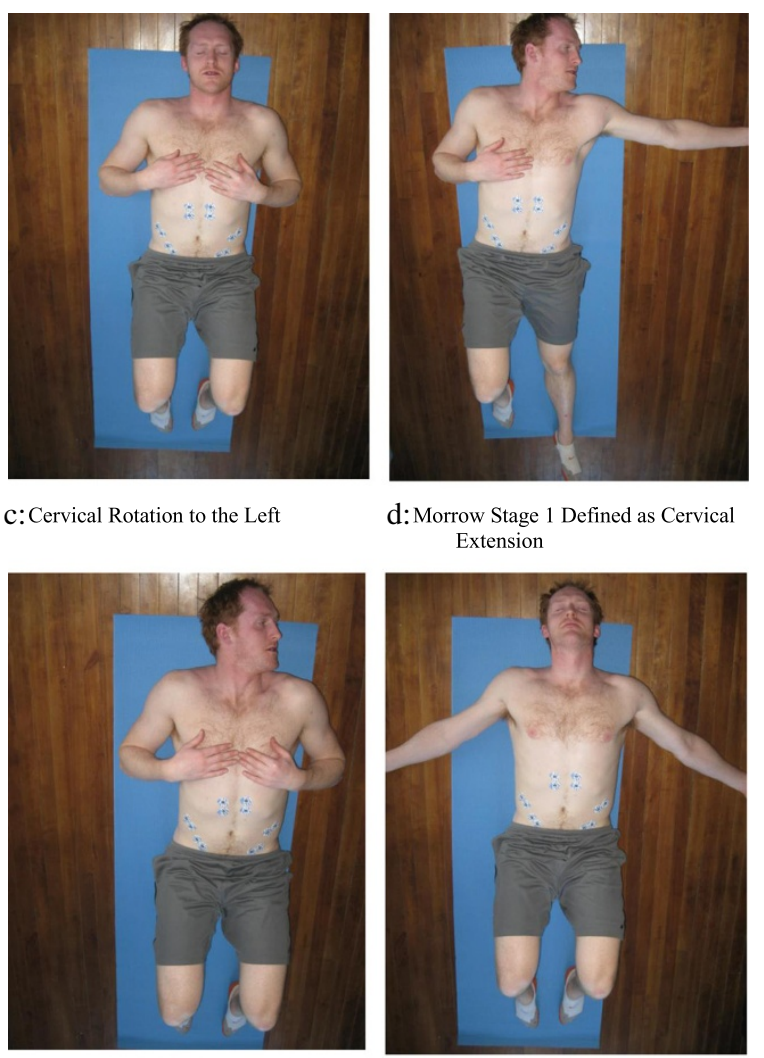

d:Morrow Stage 1 Defined as Cervical Extension

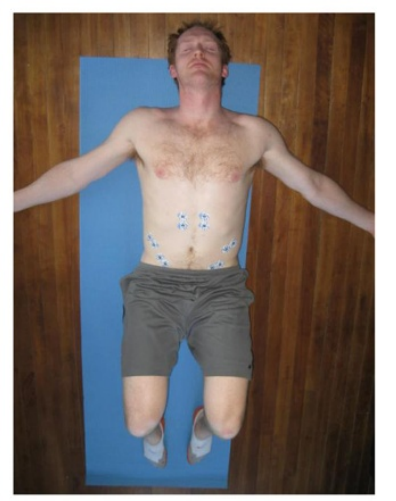

e: Morrow Stage 2 Defined as Cervical Flexion

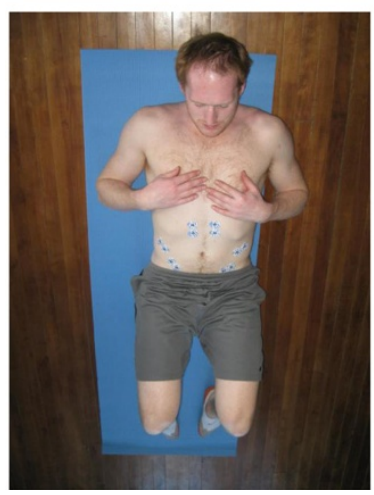

Figure 1 Electrode placement and exercise position. Acronyms: ATNR: Asymptomatic Tonic Neck Reflex. Consent was obtained from the researcher in the photos. a: Supine, b: ATNR Left, c: Cervical Rotation to the Left, $\mathbf{d}$ : Morrow Stage 1 Defined as Cervical Extension, e: Morrow Stage 2 Defined as Cervical Flexion.

was then directed through a 12 bit analog-to-digital converter (Biopac MP 100) and stored on a computer. Based on the successful procedures from previous studies $[34,35]$, EMG activity was analyzed over a 3 second period corresponding to the change in the biofeedback pressure monitor from $40-50 \mathrm{mmHg}$. Each site at each position had two successful trials, which were rectified and integrated, these two trials were averaged. The average 
integral of each muscle and exercise was normalized to the rectified integral of the same muscle during the double straight leg raise test. Raw EMG signals were visually inspected for issues such as saturation and low signal to noise ratios. These data were removed from analysis. The researcher was blinded as to which files were controls or CLBP.

After the EMG electrode set up was complete, the subject was asked to perform a double leg raise exercise, which would be used for normalization of the data. After the normalization procedure was completed, the subject was instructed on how to successfully perform the hollowing maneuver in a supine crook lying position.

\section{Double leg raise exercise}

Subjects were asked to lie supine on a bench with their hips flexed to $45^{\circ}$. On the investigators mark the subject would raise both feet $1 \mathrm{~cm}$ off a plinth and hold the position for ten seconds. This exercise was then used to normalize the exercise EMG data. A submaximal isometric contraction was performed for normalization since maximal contractions are known to be unreliable in a CLBP population [36]. The double leg raise exercise was selected because it has been shown to activate all the abdominal muscles of interest to stabilize the pelvis during the maneuver [4]. Further, a maximal contraction may cause an aggravation of symptoms in those with CLBP and since this protocol was used previously [4], it allows a more consistent comparison of the studies.

\section{Abdominal hollowing maneuver}

The abdominal hollowing maneuver as previously described [4,11-14] was performed by the subjects. Subjects would lie crook lying with a Pressure Biofeedback Unit $^{\mathrm{TM}}$ (PBU) (ProTherapy Supplies, Duluth Georgia) placed under the lordotic curve of the spine between S1 and L1 (i.e. the lumbar spine was in a neutral position) to ensure the subject was able to control for anterior pelvic tilt. The PBU was set to $40 \mathrm{mmHg}$. The test-retest reliability of the the PBU has been previously reported as 0.81 [37]. Subjects were asked to perform abdominal hollowing. They were instructed to do this through several verbal cues. The head and trunk were to remain stationary and subjects were told to not flatten their back, flex forward or push through their arms or feet. They were also told to keep breathing and not hold their breath. When the subject could successfully complete the hollowing maneuver with a slow and gradual onset and hold it for ten seconds three different times the experiment continued.

Pushing through their feet, posterior pelvic tilting or flexing the trunk are strategies in which the PBU can be changed without properly performing the abdominal hollowing maneuver, therefore these were considered an unsuccessful performance. It was determined if the subjects pushed through their feet by placing weight scales (My Weigh Scales ${ }^{\mathrm{TM}}$, CanadianWeigh, Toronto, Ontario) under their feet. Breathing was monitored visually and by a capnograph (CapnoTrainer, Better Physiology Ltd. Santa Fe, New Mexico). Trunk movement was monitored by monitoring the ASIS as well as gross and quick changes to the PBU. The abdominal hollowing was performed in a manner that would gradually bias transversus abdominis, then bias IO (while still hollowing the abdominal wall). When the pattern of execution was satisfactory as determined by the physiotherapist, the subject was then asked to perform the hollowing maneuver until they were able to change the pressure in the PBU at a steady state from 40 to $50 \mathrm{mmHg}$. The subjects would then hold this isometric activation and keep the pressure at $50 \mathrm{mmHg}$ for at least ten seconds in order to control for anterior pelvic tilt.

Next the subject would perform the hollowing maneuver in six different randomized body positions three times each for ten seconds. If the investigator noticed any problems in the execution of either the double leg raise exercise or the hollowing maneuver the subject was asked to stop given a break of thirty seconds and asked to retry the exercise for one additional repetition. Electromyographic (EMG) data were taken throughout all of the exercises. When the experimenter saw that the participants had changed the pressure from 40 to $50 \mathrm{mmHg}$ it was marked as the starting point to which EMG would be analyzed for comparison. The first three seconds of successful performance were used unless, it was noticed by the experimenter that there was a pressure change at some point in the ten second activation, in which case it was noted that a different starting point should be used for the three second EMG analysis.

\section{Simulated primitive reflexes}

The six supine body positions (Figure 1) used in the experiment were positions that attempted to mimic the orientation of the body if a specific PR was stimulated. A position similar to the ATNR was chosen because it was the most frequently observed retained PR in infants with neurological lesions [22] and adult with CLBP [28]. As well, it has the potential to influence abdominal hollowing if present. Rotating the head at least $15^{\circ}$ in either direction stimulates the ATNR. The reflex causes the limbs to which the head is pointing to extend and the contralateral limbs to flex [22]. Two positions used in this study were cervical rotation to either the left (Figure 1b) or right with the arm (side to which head is pointed) extended straight out and perpendicular to the torso and the leg (side to which head is pointed) extended. The arm on the opposite side to which the head pointed was flexed with the hand laid on the chest and the leg of the 
same side was flexed $45^{\circ}$ at both the hip and knee. Another two positions were simple cervical rotation to the left (Figure 1c) or right with their arms crossed on their chest and legs/hips flexed at $45^{\circ}$.

Another position incorporated in this study attempted to simulate the Moro reflex. This reflex is stimulated by cervical extension in the supine position and has two stages [38]. Stage one occurs immediately after cervical extension, which elicits extension and abduction of the upper extremities (Figure 1d), stage two is the return to a fetal position and involves cervical flexion (Figure 1e) along with upper extremity flexion and adduction [38]. Both stages of the cervical and upper limb aspects of the Moro were mimicked in this study. Stage one was simulated by having the subject extend at the cervical spine as far as possible without causing pain and arms abducted to approximately $60^{\circ}$ resting on the plinth (Figure 1d). Stage two was simulated by having the subject flex at the cervical spine as far as possible without causing pain as well as having the arms resting on the subject's chest (Figure 1e). When the end point of cervical flexion was achieved a triangular pad was placed under the head so it could rest at that position. If end range flexion exceeded that of the pad the subject was asked to bring their head back until it was resting on the pad, which was placed to hold the maximum amount of flexion. Hips and knees were flexed to $45^{\circ}$ for both stages.

\section{Statistical analysis}

To avoid the shortcomings of research based in nullhypothesis significance testing, magnitude-based inferences and precision of estimation were employed [39]. Magnitude-based inferences on the clinical (practical) difference in abdominal muscle activation patterns between a CLBP group and a matched control in different body positions when performing the hollowing manoeuvre.

Precision of estimated (mean) differences between the control and CLBP groups were calculated using unpaired t-tests on log-transformed data, then back-transformed and expressed with 95\% confidence limits to define the range representing the uncertainty in the true value of the (unknown) population mean. Qualitative descriptors of standardized (Cohen) effect sizes were calculated as the difference between means divided by the standard deviation of the control (supine) condition and assessed using these criteria: trivial $<0.2$, small $0.2-0.5$, moderate $0.5-0.8$, large $>0.8$ [40]. Effect sizes were also calculated on the lower and upper $95 \%$ confidence limits so the mean and variability of all dependent variables could be compared on a common metric. Effects with $95 \%$ confidence limits substantially overlapping the thresholds for small positive and negative effects (exceeding 0.2 of the standard deviation on both sides of the null) were defined as unclear. Clear small or larger effect sizes (i.e. those with $>75 \%$ likelihood of having an effect size of at least small, as calculated by a previously available spreadsheet [41] were defined as substantial [42]. This analysis was performed on the normalized data as well as a ratio of IO:RA. The IO:RA ratio has been shown to be the best representation of hollowing maneuver performance in previous research $[4,16]$ as the goal of the exercise is to emphasize activation of IO and TrA while minimizing activity of RA.

\section{Results}

\section{Between group differences for IO:RA ratio}

Using magnitude based inferences, for the left IO:RA ratio the control group would be at least $75 \%$ more likely to have a substantially greater ratio in the supine (cervical neutral position), ATNR left and right, cervical rotation to the right and cervical extension positions $(\mathrm{d}=-0.54,-0.52$, $-0.77,-0.51$ and -0.54 respectively; all "moderate") than the CLBP group. Similarly on the contralateral side it was at least $75 \%$ more likely that the control group would have a greater right IO:RA ratio than the CLBP group in the supine (cervical neutral) and ATNR left positions $(\mathrm{d}=-0.58$ and -0.91 , respectively "moderate" and "large"). A greater IO:RA ratio represents relatively less RA EMG activity (Figure 2).

\section{Between group differences for normalized site specific activation levels}

Analysis of confidence limits and effect sizes illustrated $<75 \%$ likelihood of a clinical difference between the two groups in any position for the IO or EO sites (Figure 3). There were however, likely clinical differences between groups for the RA. For the left RA it was likely that the CLBP group would have greater activation than the control in the supine (cervical neutral) $(\mathrm{d}=0.97$, "large"), ATNR left $(\mathrm{d}=0.80$, "large") and right $(\mathrm{d}=0.97$, "large"), cervical rotation to the right $(d=0.70$, "moderate") and flexion $(d=0.77$, "moderate") positions (Figure 3). For the right RA it was likely that the CLBP group would have greater activation than the control in the supine (cervical neutral) $(\mathrm{d}=$ 0.87 , "large") and cervical flexion position $(\mathrm{d}=0.59$, "moderate") (Figure 3).

Statistical significance can be inferred from the $95 \%$ confidence limits. If $95 \%$ confidence limits cross the zero, the mean must have a $\mathrm{p}>0.05$, because the lower limit is less than zero while the upper limit is greater than zero. If, however, the $95 \%$ confidence limits in the figures are both on the same side of the zero, the mean has a $\mathrm{p}<0.05$ (Figures 2 and 3 ).

\section{Discussion}

The results of this paper suggest that during the performance of the hollowing maneuver the CLBP group 


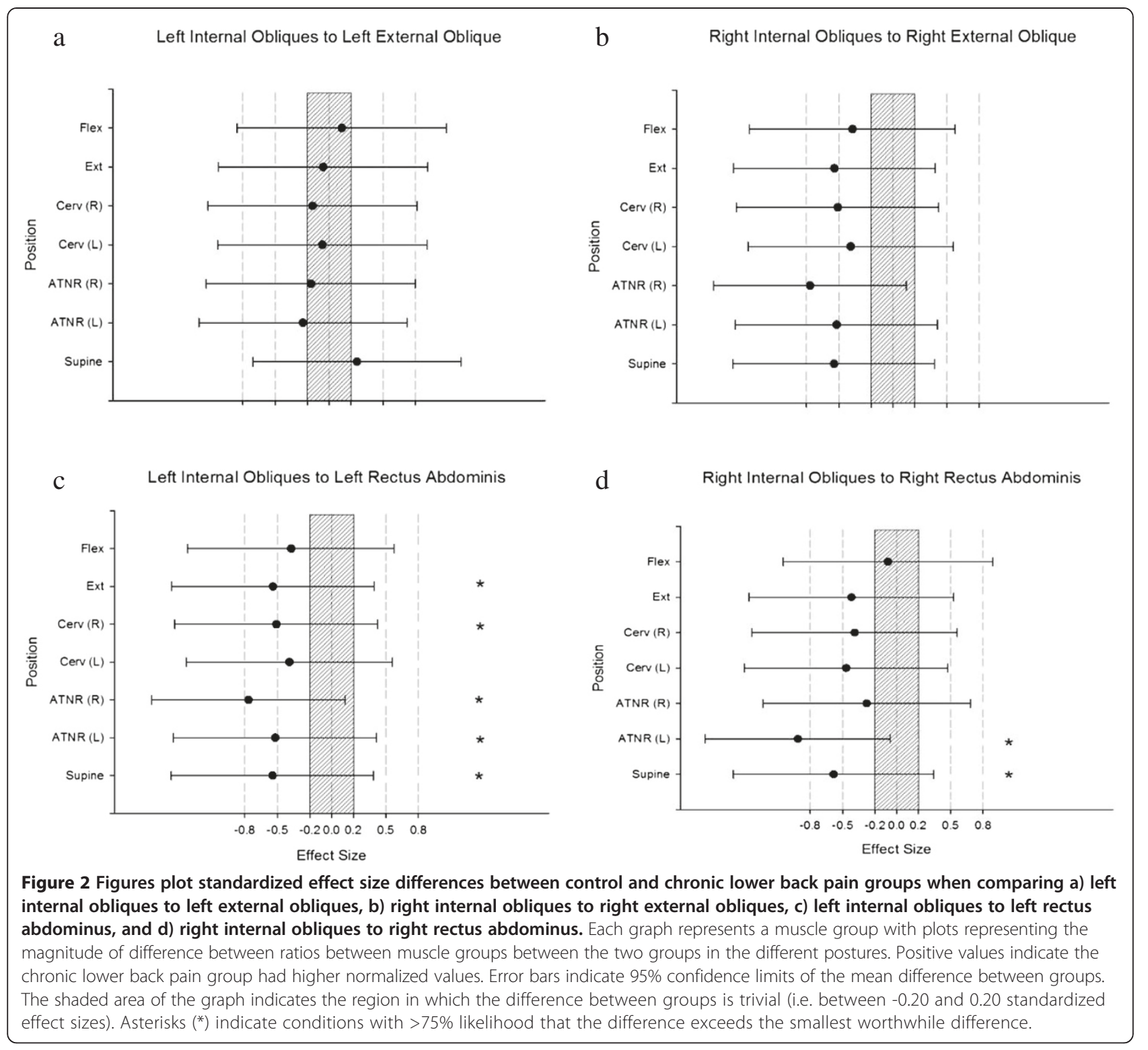

demonstrated "clinically meaningful" greater RA muscle activation levels (lower IO:RA ratios) compared to matched control groups indicating a bias toward RA when performing an abdominal hollowing exercise. As mentioned above, note that the term "clinically meaningful" indicates the observed difference is of sufficient magnitude and consistency to have at least $75 \%$ likelihood of having a substantial (meaningful) impact in a clinical setting. This indicates a spinal or supraspinal response to cervical orientation altered the activation patterns of the anterior trunk musculature.

As far as the authors are aware, this is the first study to assess the influence of altered cervical and limb position on the hollowing maneuver. These positions were intended to mimic the positions of PR. The novel finding in the present study was that activation levels were substantially affected by altered body position. This implies that in certain body positions CLBP patients illustrated an altered motor pattern when attempting to selectively activate their IO, and in other body positions had a motor pattern that clinically resembled the healthy population.

\section{Effects of cervical and limb positions on motor patterns}

While there are different abdominal musculature activation patterns between CLBP patients and healthy populations $[3,4,16,43]$, it is consistent that there is an alteration in the mechanism of how the central nervous system controls the spine $[7,44,45]$. One of the manifestations is a variable pattern of muscle substitution $[9,44]$. There are different activation patterns of the abdominal musculature during activities such as postural tasks [46], 


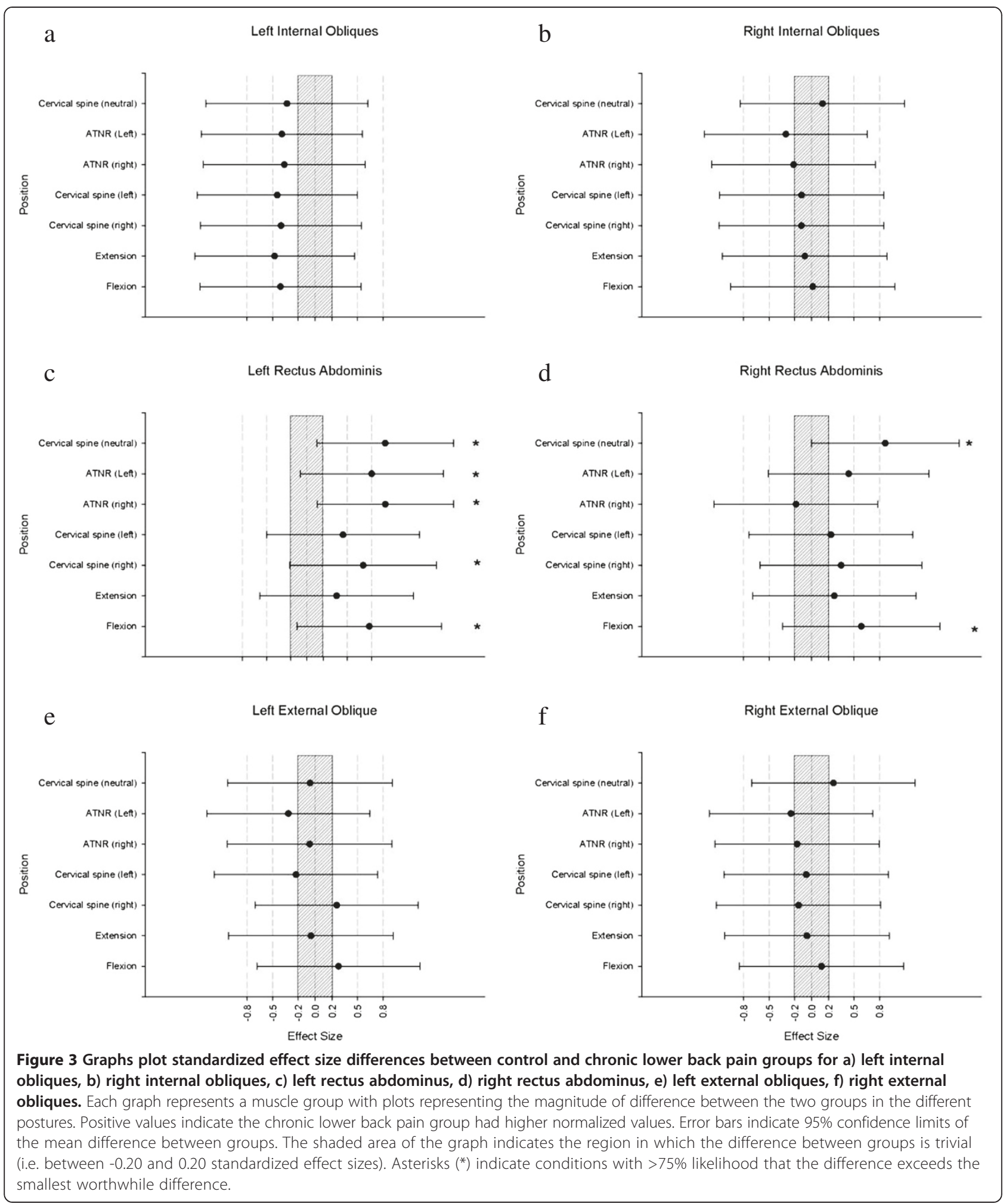

gait [47], trunk rotation [48], holding a load [49], orthopaedic tests $[50,51]$ and specific exercises [4]. Alterations in muscle activity are considered to be deficiencies in the coordination and control of the abdominal musculature and may result in a less stable spine during movement [52].

The main objective of the abdominal hollowing maneuver is to bias activation of the TrA and IO while 
minimizing activity of RA. The present study illustrated that it was clinically likely, compared to controls, for the CLBP group to have higher activation levels of the left RA in the supine (cervical neutral), ATNR left and right, cervical rotation to the right and cervical extension positions while performing the hollowing maneuver. Similarly, the CLBP group had substantially higher levels of the right RA in the cervical neutral and cervical flexion positions. This difference illustrates that when performing the hollowing maneuver in these positions the CLBP group required greater RA activation to change the pressure cuff $10 \mathrm{mmHg}$. It is thought that muscle substitution occurs because the RA attempts to compensate for the deficient IO in the CLBP group [16]. However, there is no previous research demonstrating that an alteration of body position changes the ratio of IO:RA during the hollowing maneuver.

The substantially increased level of left RA activity seen in the CLBP group was not illustrated on the right RA to the same extent. The CLBP group was clinically likely to have higher levels of left RA activity, compared to the control, in the cervical flexion, cervical rotation to the right, ATNR left/right, and cervical neutral positions. The CLBP group was clinically likely to have higher activation of the right RA, compared to the control, in only the cervical flexion and cervical neutral positions only. There are three possible explanations for this result. 1) There were a predominant number of participants with right-sided pain and thus increased contractions on the left side may have been predominant to help brace or stabilize the area. 2) Only certain PR were assessed therefore there could have been other PR or neurological soft signs (i.e. frontal release signs, clumsiness, motor incoordination, difficulty with motor sequencing) present that were not accounted for. 3) Finally the physiotherapist always stood on the right of the participant during instruction of the AHM, which may have affected the individual's focus for ipsilateral motor recruitment.

The present study did not show any clinically meaningful differences between the two groups for IO activation. This would suggest that there is not a deficiency in the IO magnitude of the CLBP group compared to the control. It is postulated that to attain the $10 \mathrm{mmHg}$ pressure change of the biofeedback cuff must require higher levels of IO activation. Therefore, there may be a ceiling effect for IO for the hollowing maneuver performed in this study. These results agree with O'Sullivan et al. $[4,16]$ who used a similar protocol. Similarly, O'Sullivan et al. $[4,16]$ did not illustrate a significant difference in activation levels of the IO between a CLBP group and healthy control. However, when a ratio of RA:IO was compared there were significant differences between groups, which indicated an altered motor program.
The CLBP group had similar activation levels of the left RA with the cervical rotation to the left and extension positions. Interestingly, these activation levels were altered by changes of cervical orientation and not the extremities. This indicates that the changes in activation were unlikely due to structural changes in the position of the muscle but more likely at the spinal and/or supraspinal level.

Other researchers have investigated the influence of altering limb position on aspects of motor control in humans. Cervical positions are known to alter the accuracy of upper limb movement in healthy people [53-55] and elbow joint position error in subjects with whiplash associated disorders [56]. The head may be used as a reference for the performance of upper limb movements and the altered proprioception of the neck may introduce error in the mechanism of central control of movement. CLBP subjects also have altered proprioception [57]. It is unknown if the trunk uses the cervical spine in a similar manner to the upper limb, however it could be possible that a similar mechanism is involved in the altered motor patterns observed here. Investigations on humans have found an influence of altering neck position on the motor system. Deutsch et al. [58] reported that head repositioning may affect the strength of the upper limb through the influence of the tonic neck reflexes. LePellec and Maton [59] concluded that the tonic neck reflexes can have a small influence on high force production with elbow flexion.

\section{CLBP-related inhibition mechanisms}

In normal function, neck receptor influences on muscle tone are involved with complex postural responses, which also reflect sensory information from visual, vestibular, proprioceptive and somatic sources [30,60]. These will interact strongly to each other and will likely have variable individual influences. Turning the head to one side accentuates the extensor tone of the limbs on that side, with flexion on the contralateral side [60]. Given the different levels of integration of PR in adults, the tone changes with repositioning of the neck can be variable and complicated.

CLBP may be associated with an overall reduction of CNS inhibition. Baliki et al. [61] theorized that the increased activation of medial pre-frontal cortex (PFC) in individuals with CLBP, compared to controls, might be due to a disruption of the mutual inhibitory interactions. A decrease in inhibition could help explain why motor cortical maps increase in volume with CLBP $[62,63]$. If CLBP causes a decrease in inhibitory interneurons then the altered activation pattern of the anterior trunk muscles of the CLBP group during the hollowing maneuver compared to the control may be due to over activity of the CNS. In terms of muscle substitution, it is generally 
thought that the RA increases its activation level to make up for a deficient ability to activate IO [16]. While theoretically and functionally this makes sense, recent research on CLBP and brain morphology and activity poses an alternate explanation. With a decrease in gray matter volume and density it is mainly a loss of inhibition that results [26]. Therefore the increased activation levels of RA may be due to an inability to inhibit this activation when attempting to perform the hollowing maneuver. This explanation would support the results in the present study as our CLBP subjects exhibited substantially higher normalized levels of both left and right RA compared to a matched control in a variety of different positions but had similar levels of IO activation. This indicates that while both groups were able to activate IO to a similar extent, however the control group was substantially better at activating IO while minimizing activity in RA.

\section{Primitive reflexes with CLBP}

While reduced inhibition helps explain muscle substitution, it does not clarify why the present study showed that altering cervical orientation can substantially affect RA activation. Subgroups of subjects with CLBP have been found to have significantly higher levels of PR than other groups [28]. PR are brainstem mediated movement patterns which are inhibited by areas in the frontal lobe [64]. PR typically start to be inhibited at six months [21] and their presence is used to assess CNS integrity [23]. While it is unknown whether the PR in this current population has resurfaced, as it does with normal advanced aging, or if they have been present throughout the subjects' life it can possibly indicate CNS disruption.

Age is an unlikely reason for the resurfaced PR as the population employed had an average age of 45 and PR re-emergence is usually not seen until the sixth decade [65]. Similarly if PR are resurfacing it would agree with the theories that there is an overall reduction in inhibition associated with reduced grey matter and CLBP $[26,61]$. If PR are present in an individual with CLBP it may be possible that the altered motor strategy is due to a reduction in supraspinal inhibition.

There was an expectation that the muscle activation patterns of the CLBP group would be more similar to the control during performance of the hollowing maneuver when placed in a position mimicking either ATNR or the supine extension reflexes (e.g. Moro reflex, tonic labyrinthine reflex $\{$ TLR $\}$ ). Placing our CLBP subjects in the ATNR position with altered position of the extremities did not substantially affect performance compared to controls. However, cervical rotation to the left with the hollowing maneuver by CLBP, had activation of left RA similar to controls. Likewise, cervical extension illustrated similar levels of RA activation between groups.
How cervical orientation affects trunk muscle activation patterns in this study can only be speculative. In this study, the subjects' body and limb orientations were not performed passively. They actively placed themselves in these positions. Hence it may be possible that the process of consciously placing the limb in a position resembling the PR is what provided inhibition.

Perhaps by inhibiting this reflex with altered head and limb orientations, it is reopening latent inhibitory synaptic pathways in the frontal lobe. This may in turn access other inhibitory pathways allowing the CLBP patients to activate IO while also inhibiting activation of RA during the hollowing maneuver. Wand et al. [66] also came to the conclusion that widespread disinhibition may be a fundamental issue with CLBP and that treatment paradigms that elicit intracortical inhibition should be explored.

\section{Limitations}

This study poses new insight into both muscular activation patterns of CLBP patients as well as how altering cervical orientation can affect these activation patterns. However, the results must be considered within the limitations of the study. In this study, only surface EMG electrodes were used. At the site of $\mathrm{IO}$, there will be recordings from TrA since it lies directly beneath this point. McGill et al. [67] reported that surface electrodes adequately represent the EMG amplitude of the deep abdominal muscles (i.e. TrA and IO) within a 15\% RMS difference. $\mathrm{Ng}$ et al. [68] indicated that electrodes placed medial to the ASIS would receive competing signals from the EO and TrA with the IO. Based on these findings, the EMG signals obtained from this abdominal location are described in the present study as the IO, which would be assumed to include EMG information from both the TrA and IO. However this limitation should not affect the interpretation of the results in this study for three reasons. 1) Anatomically it has been shown that the lower fibers of both IO and TrA have similar orientation and attachments [69]. 2) Likewise it has been proposed that they have similar synergistic functions in ipsilateral rotation and sacroiliac joint closure [70]. 3) Finally it has been shown that the hollowing maneuver is performed by the combined activity of IO and $\operatorname{TrA}$ [71]. Because of the similarity in function and anatomy, these two muscles have been recorded together with surface electrodes in a number of studies from this laboratory and their EMG activity have been differentiated from other neighbouring muscles such as the RA and EO [34-37]. A further limitation of the study was that CLBP patients without PR were not included in the investigation. In addition, palpation for the presence of activation of TrA may also have been helpful but due to the number of researcher responsibilities during the 
experiment (e.g. monitoring EMG, PBU, subject's pushing of feet, performance of hollowing maneuver and others), it was not possible to add this additional measure.

The expression of our results may not be familiar to all readers. We purposefully omitted p-values and discussion of statistical significance. While both p-values and $95 \%$ confidence limits can be used to infer statistical significance (see last paragraph of results section), 95\% confidence limits are much more information-rich to the clinician. The p-value only represents the probability of the mean response to a treatment not being zero. Concluding that a mean was "unlikely to be zero" is not a clinically useful conclusion because it does not express the variability in responses in clinically meaningful units. However, expressing results using a mean in conjunction with upper and lower confidence limits allows clinicians to easily interpret the likely effects they can expect from an intervention.

\section{Local and global abdominal hollowing}

It is clear from the descriptions used in the literature for laboratory research and clinical trials on abdominal hollowing that there are different versions of the exercise in use $[12,72,73]$. In general, there are two types of abdominal hollowing. The first aims to bias TrA over IO, EO and RA. This may be considered "local abdominal hollowing". During the hollowing maneuver described here and by O'Sullivan et al. [4,16], it should be noted that the change in the pressure biofeedback unit from $40 \mathrm{mmHg}$ to $50 \mathrm{mmHg}$ does not occur with $\operatorname{TrA}$ alone. This $10 \mathrm{mmHg}$ change requires higher levels of superficial muscle activity. Here, the goal of the exercise is to bias activation of IO while minimizing activity of RA. This may be considered "global abdominal hollowing". The former exercise is designed for translation control of individual spinal segments, while the latter is designed for movement control $[4,16]$. It should be noted that the 'global' abdominal hollowing may be performed as a progression of the 'local' abdominal hollowing or independently of it. Invariably, there will be some element of translation control with the 'global' abdominal hollowing even if it is performed independently of the 'local' abdominal hollowing. This requires further clarification, but is beyond the scope of this paper. In this study, 'global' abdominal hollowing was taught as a progression from 'local' abdominal hollowing.

The change in the PBU from $40-50 \mathrm{mmHg}$ may also consist of a contribution from a co-contraction with lumbar multifidus since this is known to co-contract with $\operatorname{TrA}$ [74]. Given that the change in the PBU depends on a change in the orientation of the posterior abdominal wall, and this may be at least be partially dependent upon intra-abdominal pressure, it may be permissible that coordination of the whole deep muscle system or cylinder (TrA, diaphragm, pelvic floor, psoas major, deep lumbar multifidus) contributes to this change [72]. Although other studies have looked at abdominal hollowing [75,76], only O'Sullivan et al. $[4,16]$ have looked at 'global' abdominal hollowing, used EMG and as a monitoring tool and standardized the amount of abdominal hollowing with a PBU.

\section{Conclusion}

Further research is required to understand the mechanism of altering cervical and limb position on abdominal activity, and the influence of other PR or neurological soft signs on CLBP. Clinically, the re-emergence or continuing presence of PR may influence muscle activation patterns of CLBP patients during the hollowing maneuver. A trained physiotherapist was able to identify the presence of PR symptoms in all the participants of the CLBP group. These results may help with the subclassification of CLBP patients. This could open up new assessment protocols for CLBP patients in which PR presence should be determined. If there are PR present then it can be possible that there is a decrease level of supraspinal inhibition and that the main goal of treatment should not be abdominal hollowing, but learning to inhibit RA when performing the hollowing maneuver. Alternatively, a treatment protocol of PR inhibition may be required especially if the patients have difficulty learning the hollowing maneuver or minimizing RA activity during the hollowing maneuver [77]. Likewise clinicians may find it easier to teach individuals how to inhibit RA by changing cervical orientation [19].

This is the first study to assess the influence of cervical and limb orientation on the hollowing maneuver. These findings should be interpreted with caution. There is some discrepancy in the literature regarding abdominal hollowing and the terminology of the different versions can get combined. There is clinical evidence to support the use of a rehabilitation program that includes 'local' abdominal hollowing in chronic and recurrent low back pain through meta-analysis [7] and articular low back pain through systematic review [78]. Although there is clinical support for 'global' abdominal hollowing [16], a systematic review or metaanalysis has not been conducted. This would provide further support for this exercise in the management of CLBP.

\section{Abbreviations}

ASIS: Anterior superior iliac spine; ATNR: Asymptomatic tonic neck reflex; CLBP: Chronic low back pain; CNS: Central nervous system;

EMG: Electromyography; EO: External obliques; IO: Internal obliques; L1: First lumbar vertebrae; PBU: Pressure biofeedback unit; PFC: Pre-frontal cortex; PR: Primitive reflexes; RA: Rectus abdominus; S1: First sacral vertebrae; SD: Standard deviation; TLR: Tonic Labyrinthine Reflex; TrA: Transversus abdominus. 


\section{Competing interests}

There were no competing or conflicting interests in the completion of this study.

\section{Authors' contributions}

All authors were involved in the idea conception and study development as well as writing and reviewing the paper. KP and SGibbons were involved in data collection. KP also provided the data analysis. All authors read and approved the final manuscript.

\section{Authors' information}

Kevin Parfrey: Mr. Parfrey completed his Master of Science (Kinesiology) degree from Memorial University of Newfoundland. He also completed a Master of Physiotherapy degree from Dalhousie University in Halifax, Nova Scotia. He presently works as a physiotherapist for KD Physical Therapies in the Halifax region.

Sean Gibbons: Mr. Gibbons is a registered certified physiotherapist and Director of SMARTERehab owner of Stability Physiotherapy in St. John's, Newfoundland, Canada. He is completing his PhD in clinical epidemiology at Memorial University of Newfoundland.

Eric Drinkwater: Dr. Drinkwater completed his Master of Physical Education degree from Memorial University of Newfoundland. He collected data for his doctoral degree at the Australian Institute of Sport in Canberra, completing his PhD through Victoria University (Melbourne) in 2006. Dr. Drinkwater presently works with School of Exercise and Health Sciences at Edit Cowan University in Perth Australia.

David Behm: Dr. Behm is the Associate Dean for Graduate Studies and Research in the School of Human Kinetics and Recreation at Memorial University of Newfoundland in St. John's, Newfoundland, Canada.

\section{Acknowledgements}

The authors wish to thank the participants for their cooperation and patience.

\section{Funding}

This research was partially funded by the Natural Science and Engineering Research Council of Canada.

\section{Author details}

School of Human Kinetics and Recreation, Memorial University of Newfoundland, St. John's, Newfoundland, Canada. 'Faculty of Medicine, Memorial University of Newfoundland, St. John's, Newfoundland, Canada. ${ }^{3}$ School of Exercise and Health Sciences, Edit Cowan University, Perth, Australia.

Received: 30 April 2013 Accepted: 12 February 2014

Published: 22 February 2014

\section{References}

1. Waddell G: The Back Pain Revolution. 2nd edition. UK: Churchill Livingstone; 2004.

2. Beith I, Synnott R, Newman S: Abdominal muscle activity during the abdominal hollowing manoeuvre in the four point kneeling and prone positions. Man Ther 2001, 6(2):82-87.

3. Hodges $P$ : Is there a role for transversus abdominis in lumbo-pelvic stability? Man Ther 1999, 4(2):74-86.

4. O'Sullivan P, Twomey L, Allison G: Altered abdominal muscle recruitment in patients with chronic back pain following a specific exercise intervention. J Orthop Sports Phys Ther 1998, 27(2):114-124.

5. Ebenbichler G, Oddsson L, Kollmitzer J, Erim Z: Sensory-motor control of the lower back: implications for rehabilitation. Med Sci Sports Exerc 2001, 33(11):1889-1898.

6. O'Sullivan P, Grahamslaw K, Kendell M, Lapenskie S, Moller N, Richards K: The effect of different standing and sitting postures on trunk muscle activity in a pain-free population. Spine 2002, 27(11):1238-1244.

7. vanDieen J, Cholewicki J, Radebold A: Trunk muscle recruitment patterns in patients with low back pain enhance the stability of the lumbar spine. Spine 2003, 28(8):834-841.

8. Macdonald D, Moseley L, Hodges P: Why do some patients keep hurting their back? Evidence of ongoing back muscle dysfunction during remission from recurrent back pain. Pain 2009, 142(3):183-188.
9. Hodges $P$, Tucker $K$ : Moving differently in pain: a new theory to explain the adaptation to pain. Pain 2011, 152:S90-S98.

10. Gibbons SGT: The role of proprioception \& sensory motor function in rehabilitation, cognitive function \& outcome prediction. In Proceedings of: The 7th National Symposium of the Kuwaiti Physical Therapy Association. Kuwait City, Kuwait; 2008:112-115.

11. Richardson C, Jull G, Toppenberg R, Comerford: Techniques for active lumbar stabilization for spinal protection: a pilot study. Aust J Physiother 1992, 38(2):105-112.

12. Gibbons S: Problems with the interpretation of abdominal hollowing in laboratory research and clinical trials: a discussion for the need of standardization and protocols. Neuromuscular Rehab Review 2014, 2:2-12.

13. Comerford M, Mottram S, Gibbons: Diagnosis of Mechanical Back Pain sub Groups \& Stability Retraining of the Lumbar Spine. Kinetic Control, Chichester, UK: Kinetic Control Movement Dysfunction Course; 2004.

14. Richardson C, Hides J, Roll S: Advanced Instruction In Retraining Motor Control For Segmental Stabilization: Realtime Ultrasound For Biofeedback, Continuing Education Course. Windsor Ontario, Canada: Human Kinetics Publishers; 1999.

15. McGill S: In Low Back Disorders: Evidence Based Prevention. 2nd edition. Edited by Robertson L, Mustain E, McCasky M, Cler C. Champaign, IL U.S.A: Human Kinetics; 2007.

16. O'Sullivan P, Twomey L, Allison G, Sinclair J, Miller K: Altered patterns of abdominal muscle activation in patients with chronic low back pain. Aust J Physiother 1997, 43(2):91-98.

17. Gibbons S: The development, initial reliability and construct validity of the motor control abilities questionnaire. Man Ther 2009, 14(S1):S22.

18. Gibbons S: Neurocognitive and sensorimotor deficits represent an important sub-classification for musculoskeletal disorders - Central Nervous System Coordination. J Icelandic Phys Ther Assoc 2011, 38(1):10-12.

19. Gibbons S: Retraining of asymmetry in recruitment of transversus abdominis. Orthop Div Rev 2008, 10:29-34.

20. Breslau N, Chilcoat H, Johnson E, Andreski P, Lucia V: Neurologic soft signs and low birthweight: their association and neuropsychiatric implications. Biol Psychiatry 2000, 47:71-79.

21. McPhillips M, Hepper P, Mulhern G: Effects of replicating primary-reflex movements on specific reading difficulties in children: a randomised, double-blind, controlled trial. Lancet 2000, 355(9203):537-541.

22. McPhillips M, Sheehy N: Prevalence of persistent primary reflexes and motor problems in children with reading difficulties. Dyslexia 2004, 10(4):316-338.

23. Zafeiriou D: Primitive reflexes and postural reactions in the neurodevelopmental examination. Pediatr Neurol 2004, 31(1):1-8.

24. Teixeira F, Alonso E, Romero V, Ortiz A, Martinez C, Otero E: Clinico-pathological correlation in dementias. J Psychiatry Neurosci 1995, 20(4):276-282.

25. VanBoxtel $M$, Bosma $H$, Jolles J, Vreeling F: Prevalence of primitive reflexes and the relationship with cognitive change in healthy adults: a report from the maastricht aging study. J Neurol 2006, 253(7):935-941.

26. Apkarian A, Sosa Y, Sonty S, Levy R, Harden R, Parrish T, Gitelman D: Chronic back pain is associated with decreased prefrontal and thalamic gray matter density. J Neurosci 2004, 24(46):10410-10415.

27. Roland M, Fairbank J: The roland-morris disability questionnaire and the oswestry disability questionnaire. Spine 2000, 25(24):3115-3124.

28. Gibbons S: Neurological soft signs are present more often and to a greater extent in adults with chronic low back pain with cognitive learning deficits. Man Ther 2009, 14(S1):S20.

29. Turk DC, Okifuji A: Pain terms and taxonomies. In Bonica's Management of Pain. 3rd edition. Edited by Loeser D, Butler SH, Chapman JJ, et al. Philadelphia (PA): Lippincott, Williams \& Wilkins; 2001:18-25. 2.

30. Von Garnier K, Koveker K, Rackwitz B, Kober U, Wilke S, Ewert T, Stucki G: Reliability of a test measuring transversus abdominus muscle recruitment with a pressure biofeedback unit. Physiotherapy 2009, 95(1):8-19.

31. Anderson $\mathrm{K}$, Behm D: Trunk muscle activity increases with unstable squat movements. Can J Appl Physiol 2005, 30(1):33-45.

32. Behm D, Anderson $K$ : The role of instability with resistance training. J Strength Cond Res 2006, 20(3):716-722.

33. Hamlyn N, Behm D, Young W: Trunk muscle activation during dynamic weight-training exercises and isometric instability activities. J Strength Cond Res 2007, 21(4):1108-1112.

34. Parfrey K, Docherty D, Workman C, Behm D: The effects of different sit- and curl-up positions on activation of abdominal and hip flexor musculature. Appl Physiol Nutr Metab 2008, 33(5):888-895. 
35. Workman C, Docherty D, Parfrey K, Behm D: Influence of pelvis position on the activation of abdominal and hip flexor muscles. J Strength Cond Res 2008, 22(5):1563-1569

36. Goddard S: Reflexes, Learning and Behavior. Eugene, Oregon. USA: Fern Ridge Press; 2005.

37. Beimborn D, Morrissey M: A review of the literature related to trunk muscle performance. Spine 1988, 13(6):655-660.

38. Allen M, Capute A: The evolution of primitive reflexes in extremely premature infants. Pediatr Res 1986, 20(12):1284-1289.

39. Hopkin W, Marshall S, Batterham A, Hanin J: Progressive statistic for studies in sports medicine and exercise science. Med Sci Sports Exerc 2009, 41(1):3-13.

40. Cohen J: Statistical power analysis for the behavioural sciences. 2nd edition. Hillsdale, New Jersey: Lawrence Erlaum Associates; 1988.

41. Hopkins W: A spreadsheet for deriving a confidence interval, mechanistic inference and clinical inference from a $p$ value. Sportscience 2007, 2007(11):16-20.

42. Liow D, Hopkins W: Velocity specificity of weight training for kayak sprint performance. Med Sci Sports Exerc 2003, 35(7):1232-1237.

43. Hodges $P$, Richardson C: Inefficient muscular stabilization of the lumbar spine associated with low back pain. A motor control evaluation of transversus abdominis. Spine 1996, 21(22):2640-2650.

44. Hodges P: Pain and motor control: from the laboratory to rehabilitation. J Electromyogr Kinesiol 2011, 21:220-228.

45. Jull G, Richardson C: Motor control problems in patients with spinal pain: a new direction for therapeutic exercise. J Manipulative Physiol Ther 2000, 23(2):115-117.

46. Hodges P, Richardson C, Jull G: Evaluation of the relationship between laboratory and clinical tests of transversus abdominis function. Physiother Res Int 1996, 1(1):30-40.

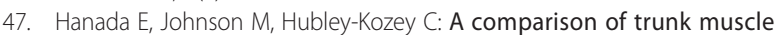
activation amplitudes during gait in older adults with and without chronic low back pain. PMR 2011, 3(10):920-928.

48. Ng K, Richardson C, Parianpour M, Kippers V: EMG activity of trunk muscles and torque output during isometric axial rotation exertion: a comparison between back pain patients and matched controls. J Orthop Res 2002, 20(1):112-121

49. Ershad N, Kahrizi S, Abadi M, Zadeh S: Evaluation of trunk muscle activity in chronic low back pain patients and healthy individuals during holding loads. J Back Musculoskelet Rehabil 2009, 22(3):165-172.

50. Hungerford B, Gilleard W, Hodges P: Evidence of altered lumbopelvic muscle recruitment in the presence of sacroiliac joint pain. Spine 2003, 28(14):1593-1600

51. Shadmehr A, Jafarian Z, Talebian S: Changes in recruitment of pelvic stabilizer muscles in people with and without sacroiliac joint pain during the active straight-leg-raise test. J Back Musculoskelet Rehabil 2012, 25(1):27-32.

52. Hodges P, van den Hoorn W, Dawson A, Cholewicki J: Changes in the mechanical properties of the trunk in low back pain may be associated with recurrence. J Biomech 2009, 42:61-66.

53. Fookson O, Smetanin B, Berkinblit M, Adamovich S, Feldman G, Poizner H: Azimuth errors in pointing to remembered targets under extreme head rotations. Neuroreport 1994, 5:885-888.

54. Guerraz M, Blouin J, Vercher J: From head orientation to hand control: evidence of both neck and vestibular involvement in hand drawing. Exp Brain Res 2003, 150:40-49.

55. Knox J, Hodges P: Changes in head and neck position affect elbow joint position sense. Exp Brain Res 2005, 165:107-113.

56. Knox J, Beilstein D, Charles S, Aarseth G, Rayer S, Treleaven J, Hodges P: Changes in head and neck position have a greater effect on elbow joint position sense in people with whiplash-associated disorders. Clin J Pain 2006, 22:512-518.

57. Brumagne S, Cordo P, Lysens R, Verschueren S, Swinnen S: The role of paraspinal muscle spindles in lumbosacral position sense in individuals with and without low back pain. Spine 2000, 25(8):989-994.

58. Deutsch H, Kilani H, Moustafa E, Hamilton N, Hebert JP: Effect of head-neck position on elbow flexor muscle torque production. Phys Ther 1987 67:517-521.

59. Le Pellec A, Maton B: The influence of tonic neck reflexes on voluntary fatiguing elbow movements in humans. Eur J Appl Physiol 1993, 67:231-238.

60. Aiello I, Rosati G, Sau GF, Patraskakis S, Bissakou M, Traccis S: Tonic neck reflexes on upper limb flexor tone in man. Exp Neurol 1988, 101(1):41-49.
61. Baliki M, Geha P, Apkarian A, Chialvo D: Beyond feeling: chronic pain hurts the brain, disrupting the default-mode network dynamics. J Neurosci 2008, 28(6):1398-1403.

62. Tsao H, Galea M, Hodges P: Reorganization of the motor cortex is associated with postural control deficits in recurrent low back pain. Brain 2008, 131:2161-2171.

63. Tsao H, Galea M, Hodges P: Driving plasticity in the motor cortex in recurrent low back pain. Eur J Pain 2010, 14(8):832-839.

64. Sudo K, Matsuyama T, Goto Y, Matsumoto A, Tashiro K: Elbow flexion response as another primitive reflex. Psychiatry Clin Neurosci 2002, 56(2):131-137.

65. Odenheimer G, Funkenstein H, Beckett L, Chown M, Pilgrim D, Evans D, Albert M: Comparison of neurologic changes in 'successfully aging' persons vs the total aging population. Arch Neurol 1994, 51(6):573-580

66. Wand B, Parkitny L, O'Connell N, Luomajoki H, Thacker M, Moseley G: Cortical changes in chronic low back pain: current state of the art and implications for clinical practice. Man Ther 2011, 16(1):15-20.

67. McGill S, Juker D, Kropf P: Appropriately placed surface EMG electrodes reflect deep muscle activity (psoas, quadratus lumborum, abdominal wall) in the lumbar spine. J Biomech 1996, 29(11):1503-1507.

68. $\mathrm{Ng} \mathrm{J}$, Kippers $\mathrm{V}$, Richardson C: Muscle fibre orientation of abdominal muscles and suggested surface EMG electrode positions. Electromyogr Clin Neurophysiol 1998, 38:51-58.

69. Urquhart D, Barker P, Hodges P, Story I, Briggs C: Regional morphology of the transversus abdominis and obliquusinternus and externus abdominis muscles. Clin Biomech 2005, 20(3):233-241.

70. Urquhart D, Hodges P: Differential activity of regions of transversus abdominis during trunk rotation. Eur Spine J 2005, 14(4):393-400.

71. Monfort-Pañego M, Vera-García J, Sánchez-Zuriaga D, Sarti-Martínez A: Electromyographic studies in abdominal exercise: a literature synthesis. J Manipulative Physiol Ther 2009, 32(3):232-244.

72. Gibbons SGT: Integrating the psoas major and deep sacral guteus maximus muscles into the lumbar cylinder model. Rome, Italy: Proceedings of: "The Spine": World Congress on Manual Therapy; 2005.

73. Gibbons S, Clark J: Specific motor control exercise for lumbo-pelvic pain of articular origin: a systematic review. Man Ther 2009, 14(S1):S16-S17.

74. Richardson C, Jull G, Hodges P. Hides J: Therapeutic exercises for spinal segmental stabilization in low back pain: scientific basis and clinical approach. London, England: Churchill Linvingstone; 1999.

75. Gorbet N, Selkow L, Joseph M, Susan S: No difference in transverse abdominis activation ratio between healthy and asymptomatic low back pain patients during therapeutic exercise. Rehabil Res Pract 2010, 18:1-6.

76. Park D, Lee $\mathrm{H}$ : Activation of abdominal muscles during abdominal hollowing in four different positions. J Phys Ther Sci 2010, 22:203-207.

77. Gibbons S: Primitive reflex inhibition and sensory motor training improves cognitive learning function and symptoms in chronic disabling low back pain: a case series. Man Ther 2009, 14(\$1):S24.

78. Byström G, Rasmussen-Barr E, Grooten W: Motor control exercises reduces pain and disability in chronic and recurrent low back pain: a meta-analysis. Spine 2013, 38(6):E350-E358.

doi:10.1186/1471-2474-15-52

Cite this article as: Parfrey et al:: Effect of head and limb orientation on trunk muscle activation during abdominal hollowing in chronic low back pain. BMC Musculoskeletal Disorders 2014 15:52.

\section{Submit your next manuscript to BioMed Central and take full advantage of:}

- Convenient online submission

- Thorough peer review

- No space constraints or color figure charges

- Immediate publication on acceptance

- Inclusion in PubMed, CAS, Scopus and Google Scholar

- Research which is freely available for redistribution 